\title{
Correction to: MobiEye: turning your smartphones into a ubiquitous unobtrusive vital sign monitoring system
}

\author{
Omkar Patil ${ }^{1} \cdot$ Wei Wang ${ }^{2} \cdot$ Yang Gao ${ }^{2} \cdot$ Zhanpeng Jin $^{2}$ \\ Published online: 12 January 2021 \\ (c) China Computer Federation (CCF) 2021

\section{Correction to: \\ CCF Transactions on Pervasive Computing and Interaction (2020) 2:97-112 \\ https://doi.org/10.1007/s42486-020-00033-3}

The article "MobiEye: turning your smartphones into a ubiquitous unobtrusive vital sign monitoring system", written by Omkar Patil · Wei Wang · Yang Gao · Zhanpeng Jin, was originally published online on 22 June 2020 with Open Access under "Creative Commons Attribution (CC BY) licence 4.0" "Creative Commons Attribution Non-Commercial (CC BY-NC 4.0)"].

After publication in volume 2 issue 2 page $97-112$ the author(s) decided to cancel the Open Access. Therefore, the copyright of the article has been changed on 22 December
2020 to (C) China Computer Federation (CCF) 2021 with all rights reserved.

Zhanpeng Jin

zjin@buffalo.edu

Omkar Patil

opatil1@binghamton.edu

Wei Wang

wwang49@buffalo.edu

Yang Gao

ygao36@buffalo.edu

1 Department of Biomedical Engineering, Binghamton University, University of New York, StateBinghamton, USA

2 Department of Computer Science and Engineering, University At Buffalo, State University of New York, Buffalo, USA 